\title{
Eine neue Bestimmung der Moleküldimensionen
}

\section{Doctoral Thesis}

Author(s):

Einstein, Albert

Publication date:

1905

Permanent link:

https://doi.org/10.3929/ethz-a-000565688

Rights / license:

In Copyright - Non-Commercial Use Permitted 


\section{EINE NEUE BESTIMMUNG DER MOLEKULDIMENSIONEN}

\section{INAUGURAL-DISSERTATION}

ZUR

ERLANGUNG DER PHILOSOPHISCHEN DOKTORWÜRDE

DER

HOHEN PHILOSOPISCHEN FAKULTÄT

(MATHEMATISCH-NATURWISSENSCHAFTLICHE SEKTION)

DER

UNIVERSITÄT ZÜRIOH

VORGELEGT

vON

\section{ALBERT EINSTEIN}

AUS ZURICH

Begutachtet von den Herren Prof. Dr. A. KLEINER. und

Prof. Dr. H. BURKHARDT

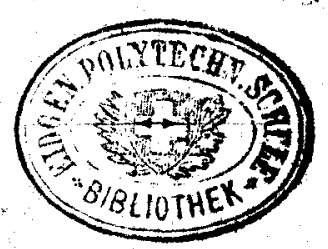

BERN

BUOHDRUCKEREI K. J. WYSS

1905 


\section{EINE NEUE BESTIMMUNG DER MOLEKÜLDIMENSIONEN}

\section{INAUGURAL-DISSERTATION \\ ZUR}

ERIANGUNG DER PHILOSOPHISCHEN DOKTORWÜRDE

DER

HOHEN PHILOSOPISCHEN FAKULTÄT

(MATIFEMATISCIL-NATUIRWISSENSCILAFTLICHI SEK'TION)

DER

UNIVERSIT ÄT ZÜRICH

VORGELEGT

VON

\section{ALBERT EINSTEIN}

AUS zÜRICII

Begutachtet von den Herren Prof. Dr. A. KLEINER und

Prof. Dr. H. BURKHARD'T

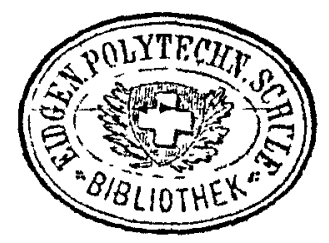

BERN

BUCHDRUCKEREI K. J. WYSS

1905 

Meinem Freunde

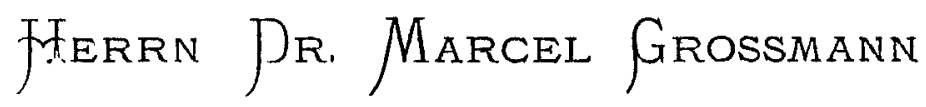

GEWIDMET 


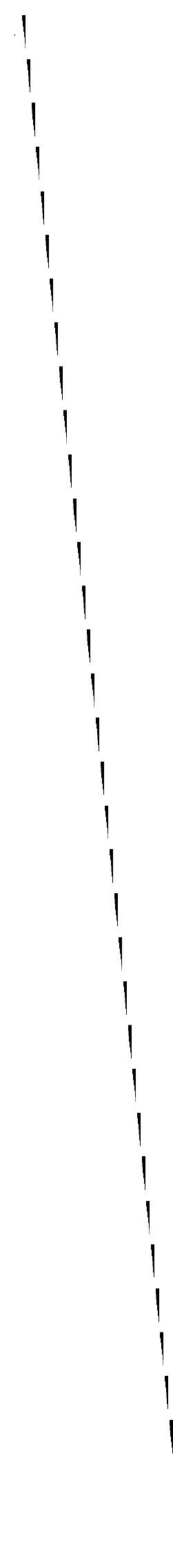




\section{Eine neue Bestimmung der Moleküldimensionen.}

Die ältesten Bestimmungen der wahren Grösse der Moleküle hat die kinetische Theorie der Gase ermöglicht, während die an Flüssigkeiten beobachteten physikalischen Phänomene bis jetzt zur Bestimmung der Molekülgrössen nicht gedient haben. Es liegt dies ohne Zweifel an den bisher unüberwindlichen Schwierigkeiten, welche der Entwickelung einer ins einzelne gehenden molekularkinetischen Theorie der Flüssigkeiten entgegenstehen. In dieser Arbeit soll nun gezeigt werden, dass man die Grösse der Moleküle des gelösten Stoff's in einer nicht dissoziierten verdünnten Lösung aus der inneren Reibung der Lösung und des reinen Lösungsmittels und aus der Diffusion des gelösten Stoffes im Lösungsmittel ermitteln kann, wenn das Volumen eines Moleküls des gelösten Stoffs gross ist gegen das Volumen eines Moleküls des Lösungsmittels. Ein derartiges gelöstes Molekül wird sich nämlich bezüglich seiner Beweglichkeit im Lösungsmittel und bezüglich seiner Beeinflussung der inneren Reibung des letzteren annähernd wie ein im Lösungsmittel suspendierter fester Körper verhalten, und es wird erlaubt sein, auf die Bewegung des Lösungsmittels in unmittelbarer Nähe eines Moleküls die hydrodynamischen Gleichungen anzuwenden, in welchen die Flüssigkeit als homogen betrachtet, eine molekulare Struktur derselben also nicht berücksichtigt wird. Als Form der festen Körper, welche die gelösten Moleküle darstellen sollen, wählen wir die Kugelform. 
§ 1. Ueber die Beeinflussung der Bewegung einer Flüssigkeit durch eine sehr kleine in derselben. suspendierte Kugel.

Es liege eine inkompressible homogene Flüssigkeit mit dem Reibungskoeffizienten $k$ der Betrachtung zugrunde, deren Geschwindigkeitskomponenten $u, v, w$ als Funktionen der Koordinaten $x, y, z$ und der Zeit gegeben seien. Von einem beliebigen Punkt $x_{0}, y_{0}, z_{0}$ aus denken wir uns dic Funktionen $u, v, w$ als Funktionen von $x-x_{0}, y-y_{0}, z-z_{0}$. nach dem Taylorschen Satze entwickelt und um diesen Punkt ein so. kleines Gebiet $G$ abgegrenzt, dass innerhalb desselben nur dielinearen Glieder dieser Entwickelung berücksichtigt werden. -müssen. Die Bewegung der in $G$ enthaltenen Flüssigkeit kann dann bekanntlich als die Superposition dreier Bewegungen aufgefasst werden, nämlich

1. einer Parallelverschiebung aller Flüssigkeitsteilchen ohne Aenderung von deren relativer Lage,

2. einer Drehung der Flüssigkeit ohne Aenderung der relativen Lage der Flüssigkeitsteilchen,

3. einer Dilatationsbewegung in drei aufeinander senkrechten. Richtungen (den Hauptdilatationsrichtungen).

Wir denken uns nun im Gebiete $G$ einen kugelförmigen starren Körper, dessen Mittelpunkt im Punkte $x_{0}, y_{0}, z_{0}$ liege und dessen Dimensionen gegen diejenigen des Gebietes $G$ sehr klein seien. Wir nehmen ferner an, dass die betrachtete Bewegung eine so. langsame sei, dass die kinetische Energie der Kugel sowie diejenige der Flüssigkeit vernachlässigt werden können. Es werde ferner angenommen, dass die Geschwindigkeitskomponenten eines Oberflächenelementes der Kugel mit den entsprechenden Geschwindigkeitskomponenten der unmittelbar benachbarten Flüssigkeitsteilchen übereinstimme, $d$. h., dass auch die (kontinuierlich gedachte) Trennungsschicht überall einen nicht unendlich kleinen Koeffizienten der inneren Reibung aufweise.

Es ist ohne weiteres klar, dass die Kugel die Teilbewegungen 1. und 2. einfach mitmacht, ohne die Bewegung der benachbarten Flüssigkeit $\mathrm{zu}$ modifizieren, da sich bei diesen Teilbewegungen die Flüssigkeit wie ein starrer Körper bewegt, und da wir die Wirkungen der Trägheit vernachlässigt haben. 
Die Bewegung 3. aber wird durch das Vorhandensein der Kugel modifiziert, und es wird unsere nächste Aufgabe sein, den Einfluss der Kugel auf diese Flüssigkeitsbewegung zu untersuchen. Beziehen wir die Bewegung 3. auf ein Koordinatensystem, dessen Achsen den Hauptdilatationsrichtungen parallel sind, und setzen wir

$$
\begin{aligned}
& x-x_{0}=\xi \\
& y-y_{0}=\eta \\
& z-z_{0}=\zeta,
\end{aligned}
$$

so lässt sich jene Bewegung, falls die Kugel nicht vorhanden ist, durch die Gleichungen darstellen:

$$
\left\{\begin{array}{l}
u_{0}=A \xi, \\
v_{0}=B \eta \\
w_{0}=C \xi ;
\end{array}\right.
$$

$A, B, C$ sind Konstanten, welche wegen der Inkompressibilität der Flüssigkeit die Bedingung erfüllen:

$$
A+B+C=0 \text {. }
$$

Refindet sich nun im Punke $x_{0}, y_{0}, z_{0}$ die starre Kugel mit dem Radius $P$, so ändert sich in der Umgebung derselben die Flüssigkeitsbewegung. Im folgenden wollen wir der Bequemlichkeit wegen $P$ als " endlich 》 bezeichnen, dagegen die Werte von $\xi, \eta, \zeta$, für welche die Flüssigkeitsbewegung durch die Kugel nicht mehr merklich modifiziert wird, als "unendlich gross".

Zunächst ist wegen der Symmetrie der betrachteten Flüssigkeitsbewegung klar, dass die Kugel bei der betrachteten Bewegung weder eine Translation noch eine Drehung ausführen kann, und wir erhalten die Grenzbedingungen:

wobei

$$
u=v=w=0 \text { für } \rho=P,
$$

$$
\rho=\sqrt{\xi^{2}+\eta^{2}+\xi^{2}}>0
$$

gesetzt ist. Hierbei bedeuten $u, v, w$ die Geschwindigkeitskomponenten der nun betrachteten (durch die Kugel modifizierten) Bewegung. Setzt man

$$
\left\{\begin{array}{c}
u=A \xi+u_{1} \\
v=B \eta+v_{1}, \\
w=C \zeta+w_{1}
\end{array}\right.
$$

so müsste, da die in Gleichungen (3) dargestellte Bewegung im Unendlichen in die in Gleichungen (1) dargestellte über- 
gehen soll, die Geschwindigkeiten $u_{1}, v_{1}, w_{1}$ im Unendlichen verschwinden.

Die Funktionen $u, v, w$ haben den Gleichungen der Hydrodynamik zu genügen unter Berücksichtigung der inneren Reibung und unter Vernachlässigung der Trägheit. Es gelten also dic Gleichungen ${ }^{1}$ )

$$
\left\{\begin{array}{c}
\frac{\delta p}{\delta \xi}=k \Delta u \frac{\delta p}{\delta \eta}=k \Delta v \frac{\delta p}{\delta \zeta}=\Delta w, \\
\frac{\delta u}{\delta \xi}+\frac{\delta v}{\delta \eta}+\frac{\delta w}{\delta \zeta}=0
\end{array}\right.
$$

wobei $\Delta$ den Operator

$$
\frac{\delta^{2}}{\delta \xi^{2}}+\frac{\delta^{2}}{\delta \eta^{2}}+\frac{\delta^{2}}{\delta \xi^{2}}
$$

und $p$ den hydrostatischen Druck bedeutet.

Da die Gleichungen (1) Lösungen der Gleichungen (4) und letztere linear sind, müssen nach (3) auch die Grössen $u_{1}, v_{1}, w_{1}$ den Gleichungen (4) genügen. Ich bestimmte $u_{1}, v_{1}, w_{1}$ und $p$ nach einer im $\S 4$ der erwähnten Kirchhoffschen Vorlesung angegebenen Methode ${ }^{2}$ ) und fand:

1) G. Kirchhoff, Vurlesungen über Mechanik. 26. Vorl.

2) Aus den Gleichungen (4) folgt $\Delta p=0$. Ist $p$ dieser Bedingung gemäss angenommen und eine Funktion $V$ bestimmt, die der Gleichung

$$
\Delta V=\frac{1}{k} p
$$

genügt, so exlüllt man die Gleichungen (4), wenn man

$$
u=\frac{\delta V}{\delta \xi}+u^{\prime}, \quad v=\frac{\delta V}{\delta \eta}+v^{\prime}, \quad w=\frac{\delta V}{\delta \zeta}+w^{\prime}
$$

setzt und $\boldsymbol{u}^{\prime}, \boldsymbol{v}^{\prime} w^{\prime}$ so wäblt, dass $\Delta u^{\prime}=0, \Delta v^{\prime}=0$ und $\Delta w^{\prime}=0$ und

ist. »

$$
\frac{\delta u^{\prime}}{\delta \xi}+\frac{\delta v^{\prime}}{\delta \eta}+\frac{\delta w^{\prime}}{\delta \zeta}=-\frac{1}{k} p
$$

Setzt man nun

und im Einklang hiermit

$$
\frac{p}{k}=2 c \frac{\hat{\delta}^{2} \frac{1}{p}}{\delta \xi^{2}}
$$

und

$$
V=c \frac{\delta^{2} p}{\delta^{2} \xi^{3}}+b \frac{\delta^{2} \frac{1}{p}}{\delta \xi^{2}}+\frac{a}{2}\left(\xi^{2}-\frac{\eta^{2}}{2}-\frac{\zeta^{2}}{2}\right)
$$




$$
\begin{aligned}
& -9- \\
& (5)\left\{\begin{array}{l}
p=-\frac{5}{3} k P^{3}\left\{A \frac{\delta^{2}\left(\frac{1}{\rho}\right)}{\delta \xi^{2}}+B \frac{\delta^{2}\left(\frac{1}{p}\right)}{\delta \eta^{2}}+C \frac{\delta^{2}\left(\frac{1}{\delta}\right)}{\delta \zeta^{2}}\right\}+\text { konst., } \\
u=A \xi-\frac{5}{3} P^{3} A \frac{\xi}{\rho^{3}}-\frac{\delta D}{\delta \xi}, \\
v=B \eta-\frac{5}{3} P^{3} B \frac{\eta}{\rho^{3}}-\frac{\delta D}{\delta \eta} \\
w=C \zeta-\frac{5}{3} P^{3} C \frac{\zeta}{\rho^{8}}-\frac{\delta D}{\delta \zeta},
\end{array}\right.
\end{aligned}
$$

wobei

(5 a)

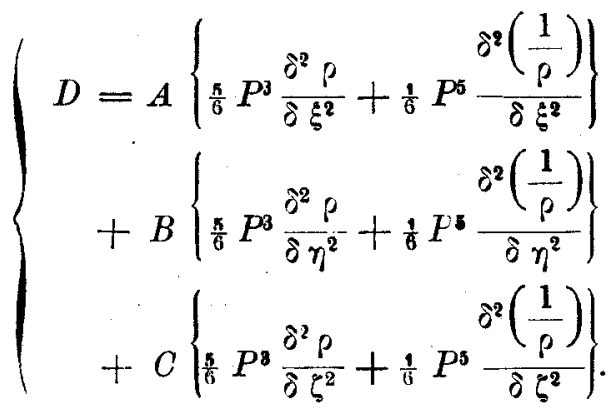

Es ist leicht $\mathrm{zu}$ beweisen, dass die Gleichungen (5) Lösungen der Gleichungen (4) sind. Denn da

$$
\Delta \xi=0, \Delta \cdot \frac{1}{\rho}=0, \Delta \rho=\frac{2}{p}
$$

und

erhält man

$$
\Delta\left(\frac{\xi}{\rho^{3}}\right)=-\frac{\delta}{\delta \xi}\left\{\Delta\left(\frac{1}{\rho}\right)\right\}=0,
$$

$k \Delta u=-k \frac{\delta}{\delta \xi}(\Delta D)=-k \frac{\delta}{\delta \xi}\left\{\frac{\delta^{2} \frac{1}{\rho}}{\partial} P^{3} A \frac{\rho}{\delta \xi^{2}}+\frac{5}{3} P^{3} B \frac{\delta^{2} \frac{1}{P}}{\delta \eta^{2}}+\ldots\right\}$.

$$
u^{\prime}=-2 c \frac{\partial \frac{1}{\partial}}{\delta \xi}, \quad v^{\prime}=\text { C. } \quad w^{\prime}=0,
$$

so lassen sich die Konstanten $a, b, c$ so bestimmen, dass für $p=P \quad u=v$ $=v=0$ ist. Durch Superposition dreier derartiger Lösungen erhält man die in den Gleichungen (5) und (5 a) angegebene Lobsung. 
Der zuletzt erhaltene Ausdruck ist aber nach der ersten der Gleichungen (5) mit $\frac{\delta n}{\delta \xi}$ identisch. Auf gleiche Weise zeigt man, dass die zweite und dritte der Gleichungen (4) erfüllt ist. Ferner erhält man

$$
\begin{aligned}
& \frac{\delta u}{\delta \xi}+\frac{\delta v}{\delta \eta}+\frac{\delta w}{\delta \xi}=(A+B+C) \\
& \quad+\frac{\delta}{3} P^{3}\left\{A \frac{\delta^{2}\left(\frac{1}{p}\right)}{\delta \xi^{2}}+B \frac{\delta^{2}\left(\frac{1}{p}\right)}{\delta \eta^{2}}+C \frac{\delta^{2}\left(\frac{1}{p}\right)}{\delta \zeta^{2}}\right\}-\Delta D .
\end{aligned}
$$

Da aber nach Gleichung (5a)

$$
\Delta D=\frac{\delta}{3} A P^{3}\left\{A \frac{\delta^{2}\left(\frac{1}{\rho}\right)}{\delta \xi^{2}}+B \frac{\delta^{2}\left(\frac{1}{\rho}\right)}{\delta \eta^{2}}+C \frac{\delta^{2}\left(\frac{1}{\rho}\right)}{\delta \zeta^{2}}\right\},
$$

so folgt, dass auch die letzte der Gleichungen (4) erfüllt ist. Was die Grenzbedingungen betrifft, so gehen zunächst für unendlich grosse $\rho$ unsere Gleichungen für $u, v, w$ in die Gleichungen (1) über. Durch Einsetzen des Wertes von $D$ aus Gleichung (5a) in die zweite der Gleichungen (5) erhält man:

$$
\left\{\begin{array}{l}
u=A \xi-\frac{5}{2} \frac{P^{3}}{\rho^{6}} \xi\left(A \xi^{2}+B \eta^{2}+C \xi^{2}\right) \\
\quad+\frac{\kappa}{2} \frac{P^{5}}{\rho^{7}} \xi\left(A \xi^{2}+B \eta^{2}+C \zeta^{2}\right)-\frac{P^{5}}{\rho^{5}} A \xi .
\end{array}\right.
$$

Man erkennt, dass $u$ für $p=P$ verschwindet. Gleiches gilt aus Symmetriegründen für $v$ und $w$. Es ist nun bewiesen, dass durch die Gleichungen (5) sowohl den Gleichungen (4) als auch den Grenzbedingungen der Aufgabe Genüge geleistet ist.

Es lässt sich auch beweisen, dass die Gleichungen (5) die einzige mit den Grenzbedingungen der Aufgabe verträgliche Lösung der Gleichungen (4) sind. Der Beweis soll hier nur angedeutet werden. Es mögen in einem endlichen Raume die Geschwindigkeitskomponenten $u, v, w$ einer Flüssigkeit den Gleichungen (4) genügen. Existierte noch eine andere Lösung $U, V, W$ der Gleichungen (4), bei welcher an den Grenzen des betrachteten Raumes $U=u, V=v, W=w$ ist, so ist ( $U-u$, $V-v, W-w)$ eine Lösung der Geichungen (4), bei welcher die Geschwindigkeitskomponenten an der Grenze des Raumes verschwinden. Der in dem betrachteten Raume befindlichen Flüssigkeit wird also keine mechanische Arbeit zugeführt. Da wir die lebendige Kraft der Flüssigkeit vernachlässigt haben, 
so folgt daraus, dass auch die im betrachteten Raume in Wärme verwandelte Arbeit gleich Null ist. Hieraus folgert man, dass im ganzen Raume $u=u_{1}, v=v_{1}, w=w_{1}$ sein muss, falls der Raum wenigstens zum Teil durch ruhende Wände begrenzt ist. Durch Grenzübergang kann dies Resultat auch auf den Fall ausgedehnt werden, dass, wie in dem oben betrachteten Falle, der betrachtete Raum unendlich ist. Man kann so dartun, dass die oben gefundene Lösung die einzige Lösung der Aufgabe ist.

Wir legen nun um den Punkt $x_{0}, y_{0}, z_{0}$ eine Kugel vom Radius $R$, wobei $R$ gegen $P$ unendlich gross sei, und berechnen die Energie, welche in der innerhalb der Kugel befindlichen Flüssigkeit (in der Zeiteinheit) in Wärme verwandelt wird. Diese Energie $W$ ist gleich der der Flüssigkeit mechanisch zugeführten Arbeit. Bezeichnet man die Komponenten des auf die Oberfläche der Kugel vom Radius $R$ ausgeübten Druckes mit $X_{n}, Y_{n}, Z_{n}$, so ist:

$$
W=\int\left(X_{\mathrm{n}} u+Y_{\mathrm{n}} v+Z_{\mathrm{n}} v\right) d s,
$$

wobei das Integral über die Oberfläche der Kugel vom Radius $R$ $\mathrm{zu}$ erstrecken ist. Hierbei ist:

$$
\begin{aligned}
& X_{\mathrm{n}}=-\left(X \xi \frac{\xi}{\rho}+X \eta \frac{\eta}{\rho}+X \zeta \frac{\zeta}{\rho}\right), \\
& Y_{\mathrm{n}}=-\left(X \xi \frac{\xi}{\rho}+Y_{\eta} \frac{\eta}{\rho}+Y \zeta \frac{\zeta}{\rho}\right), \\
& Z_{\mathrm{n}}=-\left(Z \xi \frac{\xi}{\rho}+Z_{\eta} \frac{\eta}{\rho}+Z \zeta \frac{\zeta}{\rho}\right),
\end{aligned}
$$

wobei

$$
\begin{array}{ll}
X_{\xi}=p-2 k \frac{\delta u}{\delta \xi}, & Y_{\zeta}=Z_{\eta}=-k\left(\frac{\delta v}{\delta \zeta}+\frac{\delta w}{\delta \eta}\right), \\
Y_{\eta}=p-2 k \frac{\delta v}{\delta \eta}, & Z_{\xi}=X_{\zeta}=-k\left(\frac{\delta w}{\delta \xi}+\frac{\delta u}{\delta \zeta}\right), \\
Z_{\zeta}=p-2 k \frac{\delta w}{\delta \zeta}, & X_{\eta}=Y_{\xi}=-k\left(\frac{\delta u}{\delta \eta}+\frac{\delta v}{\delta \xi}\right) .
\end{array}
$$

Die Ausdrücke für $u, v, w$ vereinfachen sich, wenn wir beachten, dass für $\rho=R$ die Glieder mit dem Faktor $P^{\delta} / \rho^{5}$ gegenüber denen mit dem Faktor $P^{a} / \rho^{8}$ verschwinden. Wir haben zu setzen: 
(6 a)

$$
\left\{\begin{array}{c}
u=A \xi-\frac{5}{2} P^{3} \frac{\xi\left(A \xi^{2}+\mathbf{B} \eta^{2}+C \zeta^{2}\right)}{\rho^{5}}, \\
v=B \eta-\frac{5}{2} P^{3} \frac{\eta\left(A \xi^{2}+B \eta^{2}+C \zeta^{2}\right)}{\rho^{3}} . \\
w=C \zeta-\frac{5}{2} P^{3} \frac{\zeta\left(A \xi^{2}+B \eta^{2}+C \zeta^{2}\right)}{\rho^{3}} .
\end{array}\right.
$$

Für $p$ erhalten wir aus der ersten der Gleichungen (5) durch die entsprechenden Vernachlässigungen

Wir erhalten zunächst:

$$
p=-5 k P^{3} \frac{A \xi^{2}+B \eta^{2}+C \zeta^{2}}{p^{3}}+\text { konst. }
$$

$$
\begin{aligned}
& X_{\xi}=-2 k A+10 k P^{3} \frac{A \xi^{2}}{\rho^{3}}-25 k P^{3} \frac{\xi^{2}\left(A \xi^{2}+B \eta^{2}+C \zeta^{2}\right)}{\rho^{7}}, \\
& X_{\eta}=\quad+10 k P^{8} \frac{A \xi \eta}{\rho^{5}}-25 k P^{3} \frac{\eta^{2}\left(A \xi^{2}+B \eta^{2}+C \zeta^{2}\right)}{\rho^{7}} \\
& X_{\zeta}=\quad+10 k P^{3} \frac{A \xi \zeta}{\rho^{j}}+25 k P^{3} \frac{\zeta^{2}\left(A \xi^{2}+B \eta^{2}+C \zeta^{2}\right)}{\rho^{7}} \\
& \text { und hieraus }
\end{aligned}
$$

$$
X_{n}=2 A k \frac{\xi}{\rho}-10 A k P^{3} \frac{\xi}{\rho^{4}}+25 k P^{3} \frac{\xi\left(A \xi^{2}+B \eta^{2}+C \zeta^{2}\right)}{\rho^{6}} \text {. }
$$

Mit Hilfe der durch zyklische Vertauschung abzuleitenden Ausdrücke für $Y_{n}$ und $Z_{n}$ erhält man unter Vernachlässigung aller Glieder, die das Verhältnis $P / \rho$ in einer höheren als der dritten Potenz enthalten:

$$
\begin{aligned}
X_{\mathrm{n}} u+Y_{\mathrm{n}} v & +Z_{\mathrm{n}} w+\frac{2 k}{\rho}\left(A^{2} \xi^{2}+B^{2} \eta^{2}+C^{2} \xi^{2}\right) \\
& -10 k \frac{p^{3}}{\rho^{4}}\left(A^{2} \xi^{2}+.+.\right)+20 k \frac{P^{3}}{\rho^{6}}\left(A \xi^{2}+.+\right)^{2} .
\end{aligned}
$$

Integriert man über die Kugel und berücksichtigt, dass

$$
\begin{aligned}
& \int d s=4 R^{2} \pi \\
& \int \xi^{2} d s=\int \eta^{2} d s=\int \zeta^{2} d s=\frac{4}{3} \pi R^{4} \\
& \int \xi^{4} d s=\int \eta^{4} d s=\int \zeta^{4} d s=\frac{4}{5} \pi R^{6} \\
& \int \eta^{2} \zeta^{2} d s=\int \zeta^{2} \xi^{2} d s=\int \xi^{2} \eta^{2} d s={ }_{15}^{4} \pi R^{6} \\
& \int\left(A \xi^{2}+B \eta^{2}+C \zeta^{2}\right)^{2} d s={ }_{15}^{4} \pi R^{6}\left(A^{2}+B^{2}+C^{2}\right)
\end{aligned}
$$


so erhält man :

(7)

$$
W=\frac{8}{3} \pi R^{3} k \delta^{2}-\frac{8}{3} \pi P^{3} k \delta^{2}=2 \delta^{2} k(V-\Phi),
$$

wobei

und

$$
\begin{gathered}
\delta=A^{2}+B^{2}+C^{2}, \\
\quad \text { s } \pi R^{3}=V
\end{gathered}
$$

$$
\frac{4}{3} \pi P^{3}=\Phi
$$

gesetzt ist. Wäre die suspendierte Kugel nicht vorhanden $(\Phi=0)$, so. erhielte man für die im Volumen $V$ verzehrte Energie

$$
W_{u}=2 \delta^{2} k V \text {. }
$$

Durch das Vorhandensein der Kugel wird also die verzehrte Energie um $2 \delta^{2} k \Phi$ verkleinert. Es ist bemerkenswert, dass. der Einfluss der suspendierten Kugel auf die Grösse der verzehrten Energie gerade so gross ist, wie er wäre, wenn durch. die Anwesenheit der Kugel die Bewegung der sie umgebenden Flüssigkeit gar nicht modifiziert würde.

\section{§ 2. Berechnung des Reibungskoeffizienten einer} Flüssigkeit, in welcher sehr viele kleine Kugeln in regelloser Verteilung suspendiert sind.

Wir haben im vorstehenden den Fall betrachtet, dass in einem Gebiete $G$ von der oben definierten Grössenordnung eine relativ zu diesem Gebiete sehr kleine Kugel suspendiert ist und untersucht, wie dieselbe die Flüssigkeitsbewegung beeinflusst. Wir wollen nun annehmen, dass in dem Gebiete $G$ unendlich viele Kugeln von gleichem, und zwar so kleinem Radius regellos verteilt sind, dass das Volumen aller Kugeln zusammen sehr klein sei gegen das Gebiet $G$. Die Zahl der auf die Volumeneinheit entfallenden Kugeln sei $n$, wobei $n$ allenthalben in der Flüssigkeit bis auf Vernachlässigbares konstant sei.

Wir gehen nun wieder aus von einer Bewegung einer homogenen Flüssigkeit ohne suspendierte Kugeln und betrachten wieder die allgemeinste Dilatationsbewegung. Sind keine Kugeln vorhanden, so können wir bei passender Wahl des Koordinatensystems die Geschwindigkeitskomponenten $u_{0}, v_{0}, w_{0}$ in dem beliebigen Punkte $x, y, z$ des Gebietes $G$ darstellen durch die Gleichungen : 


$$
\begin{aligned}
& -14- \\
& u_{0}=A x, \\
& v_{0}=B y, \\
& w_{0}=C z
\end{aligned}
$$

wobei

$$
A+B+C=0 .
$$

Eine im Punkte $x_{v}, y_{v}, z_{v}$ suspendierte Kugel beeinflusst nun diese Bewegung in der aus Gleichung (6) ersichtlichen Weise. $\mathrm{Da}$ wir den mittleren Abstand benachbarter Kugeln als sehr gross gegen deren Radius, wäblen, und folglich die von allen suspendierten Kugeln zusammen herrührenden zusätzlichen Geschwindigkeitskomponenten gegen $u_{0}, v_{0}, w_{0}$ sehr klein sind, so erhalten wir für die Geschwindigkeitskomponenten $u, v, w$ in der Flüssigkeit unter Berücksichtigung der suspendierten Kugeln und unter Vernachlässigung von Gliedern höherer Ordnungen :

$$
\begin{aligned}
& \left(u=A x-\sum\left\{\frac{P^{3}}{\rho_{\nu}^{2}} \frac{\xi_{\nu}\left(A \xi_{\nu}^{2}+B \eta_{\nu}^{2}+C \zeta_{\nu}^{2}\right)}{\rho_{\nu}^{3}}\right.\right. \\
& \left.-\frac{5}{2} \frac{P^{5}}{P_{\nu}^{4}} \frac{\xi_{\nu}\left(A \xi_{\nu}^{2}+B \eta_{\nu}^{2}+C \zeta^{2}\right)}{P_{\nu}^{3}}+\frac{P^{s} A \xi_{\nu}}{P_{\nu}^{4}} \frac{P_{\nu}}{P_{y}}\right\}, \\
& v=B y-\sum\left\{\frac{p^{3}}{\rho_{y}^{2}} \frac{\eta_{\nu}\left(A \xi_{\nu}^{2}+B \eta_{\nu}^{2}+C \xi_{\nu}^{2}\right)}{P_{y}^{3}}\right.
\end{aligned}
$$

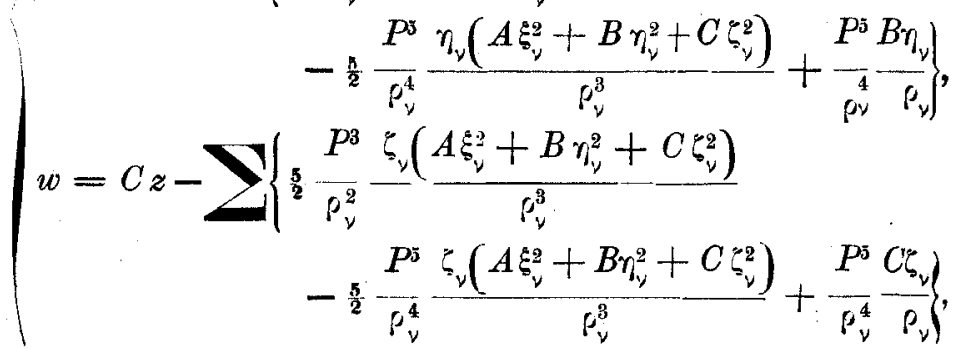

wobei die Summation über alle Kugeln des Gebietes $G$ zu erstrecken ist und

$$
\begin{aligned}
& \xi_{\nu}=x-x_{\nu}, \\
& \eta_{\nu}=y-y_{\nu}, \quad \rho_{\nu}=\sqrt{\xi_{\nu}^{2}+\eta_{\nu}^{2}+\xi_{\nu}^{2}}, \\
& \xi_{\nu}=z-z_{\nu},
\end{aligned}
$$

gesetzt ist. $x_{\nu}, y_{\nu}, z_{\nu}$ sind die Koordinaten der Kugelmittelpunkte. Aus den Gleichungen (7) und (7 a) schliessen wir ferner, 
dass die Anwesenheit jeder der Kugeln bis auf unendlich Kleines höherer Ordnung eine Verringerung der Wärmeproduktion pro Zeiteinheit um $2 \delta^{2} k \Phi$ zum Gefolge hat und dass im Gebiete $G$ die pro Volumeneinheit in Wärme verwandelte Energie den Wert hat:

oder

$$
W=2 \delta^{2} k-2 n \delta^{2} k \Phi
$$

$$
W=2 \delta^{2} k(1-\varphi)
$$

wobei $\varphi$ den von den Kugeln eingenommenen Bruchteil des Volumens bedeutet.

Gleichung (7 b) erweckt den Anschein, als ob der Reibungskoeffizient der von uns betrachteten inhomogenen Mischung von Flüssigkeit und suspendierten Kugeln (im folgenden kurz „Mischung" genannt) kleiner sei als der Reibungskoeffizient $k$ der Flüssigkeit. Dies ist jedoch nicht der Fall, da $A, B, C$ nicht die Werte der Hauptdilatationen der in Gleichungen (8) dargestellten Flüssigkeitsbewegung sind; wir wollen die Hauptdilatationen der Mischung $A^{*}, B^{*}, C^{*}$ nennen. Aus Symmetriegründen folgt, dass die Hauptdilatationsrichtungen der Mischung den Richtungen der Hauptdilatationen $A, B, C$, also den Koordinatenrichtungen parallel sind. Schreiben wir die Gleichungen (8) in der Form:

$$
\begin{aligned}
& u=A x+\sum u_{\nu}, \\
& v=B y+\sum_{\nu}, \\
& w=C z+\sum w_{\nu},
\end{aligned}
$$

so erhalten wir:

$$
A^{*}=\left(\begin{array}{l}
\delta u \\
\delta x
\end{array}\right)_{x=0}=A+\sum\left(\frac{\delta: u_{\nu}}{\delta x}\right)_{x=0}=A-\sum\left(\frac{\delta u_{\nu}}{\delta x_{\nu}}\right)_{x=0} .
$$

Schliessen wir die unmittelbaren Umgebungen der einzelnen Kugeln von der Betrachtung aus, so können wir die zweiten und dritten Glieder der Ausdrücke von $u, v, w$ weglassen und erhalten für $x=y=z=0$ : 
(9)

$$
\left\{\begin{array}{c}
-16- \\
u_{\nu}=-\frac{5}{2} \frac{P^{3}}{r_{\nu}^{2}} \frac{x_{\nu}\left(A x_{\nu}^{2}+B y_{\nu}^{2}+C z_{\nu}^{2}\right)}{r_{\nu}^{3}}, \\
v_{\nu}=-\frac{5}{2} \frac{P^{3}}{r_{\nu}^{2}} \frac{y_{\nu}\left(A x_{\nu}^{2}+B y_{\nu}^{2}+C z_{\nu}^{2}\right)}{r_{\nu}^{3}}, \\
w_{\nu}=-\frac{5}{2} \frac{P^{3}}{r_{\nu}^{2}} \frac{x\left(A x_{\nu}^{2}+B y_{\nu}^{2}+C z_{\nu}^{2}\right)}{r_{\nu}^{3}},
\end{array}\right.
$$

wobei

$$
r_{\nu}=\sqrt{x_{\nu}^{1}+y_{\nu}^{2}+z_{\nu}^{2}}>0
$$

gesetzt ist. Die Summierung erstrecken wir über das Volumen einer Kugel $K$ von sehr grossem Radius $R$, deren Mittelpunkt im Koordinatenursprung liegt. Betrachten wir ferner die regellos verteilten Kugeln als gleichmässig verteilt und setzen an Stelle der Summe ein Integral, so erhalten wir:

$$
\begin{gathered}
A^{*}=A-n \int_{K}^{\delta u_{\nu}} \frac{x_{\nu}}{\delta x_{\nu}} d y_{\nu} d z_{\nu}, \\
=A-n \int \frac{u_{\nu} x_{\nu}}{r_{\nu}} d s,
\end{gathered}
$$

wobei das letzte Integral über die Oberfläche der Kugel $K$ zu erstrecken ist. Wir finden unter Berücksichtigung von (9):

$$
\begin{aligned}
A^{*} & =A-\frac{2}{2} \frac{P^{3}}{R^{6}} n \int x_{0}^{2}\left(A x_{0}^{2}+B y_{0}^{2}+C z_{0}^{2}\right) d s, \\
& =A-n\left(\frac{4}{3} F^{3} \pi\right) A=A(1-\varphi) .
\end{aligned}
$$

Analog ist

Setzen wir

$$
\begin{aligned}
& B^{*}=B(1-\varphi), \\
& C^{*}=C(1-\varphi) .
\end{aligned}
$$

$$
\delta^{* 2}=A^{* 2}+B^{* 2}+C^{* 2}
$$

so ist bis auf unendlich Kleines höherer Ordnung:

$$
\delta^{* 2}=\delta^{2}(1-2 \varphi) \text {. }
$$

Wir haben für die Wärmeentwicklung pro Zeit- und Volumeneinheit gefunden:

$$
W^{*}=2 \delta^{2} k(1-\varphi) \text {. }
$$


Bezeichnen wir mit $k^{*}$ den Reibungskoeffizienten des Gemisches, so ist:

$$
W^{*}=2 \delta^{* 2} k^{*} .
$$

Aus den drei letzten Gleichungen erhält man unter Vernachlässigung von unendlich Kleinem höherer Ordnung:

$$
k^{*}=k(1+\varphi) \text {. }
$$

Wir erhalten also das Resultat:

Werden in einer Flüssigkeit sehr kleine starre Kugeln suspendiert, so wächst dadurch der Koeffizient der inneren Reibung um einen Bruchteil, der gleich ist dem Gesamtvolumen der in der Volumeneinheit suspendierten Kugeln, vorausgesetzt, dass dieses Gesamtvolumen sehr klein ist.

\section{3. Ueber das Volumen einer gelösten Substanz,} deren Molekularvolumen gross ist gegenüber dem des Lösungsmittels.

Es liege eine verdünnte Lösung vor eines Stoffes, welcher in der Lösung nicht dissoziiert. Ein Molekül des gelösten Stoffes sei gross gegenüber einem Molekül des Lösungsmittels und werde als starre Kugel vom Radius $P$ aufgefasst. Wir können dann das in $\S 2$ gewonnene Resultat anwenden. Bedeutet $k^{*}$ den Reibungskoeffizienten der Lösung, $k$ denjenigen des reinen Lösungsmittels, so ist:

$$
\frac{k^{*}}{k}=1+\varphi
$$

wobei $\varphi$ das Gesamtvolumen der in Lösung befindlichen Molekuile pro Volumeneinheit ist.

Wir wollen $\varphi$ für eine 1 proz. wässerige Zuckerlösung berechnen. Nach Beobachtungen von Burkhard (Tabellen von Landolt und Börnstein) ist bei einer 1 proz. wässerigen Zuckerlösung $k^{*} / k=1,0245$ (bei $20^{\circ} \mathrm{C}$ ), also $\varphi=0,0245$ für (beinahe genau) $0,01 \mathrm{~g}$ Zucker. Ein Gramm in Wasser gelöster Zucker hat also auf den Reibungskoeffizienten denselben Einfluss wie kleine suspendierte starre Kugeln vom Gesamtvolumen $2,45 \mathrm{~cm}^{\circ}$. Bei dieser Betrachtung ist der Einfluss des dem gelösten Zucker entsprechenden osmotischen Druckes auf die innere Reibung des Lösungsmittels vernachlässigt. 
Es ist nun daran zu erinnern, dass $1 \mathrm{~g}$ festen Zuckers das Volumen $0,61 \mathrm{~cm}^{3}$ besitzt. Dasselbe Volumen findet man auch für das spezifische Volumen $s$ des in Lösung befindlichen Zuckers, wenn man die Zuckerlösung als eine Mischung von Wasser und Zucker in gelöster Form auffasst. Die Dichte einer 1 proz. wässerigen Zuckerlösung (bezogen auf Wasser von derselben Temperatur) bei $17,5^{\circ}$ ist nämlich 1,00388. Man hat also (unter Vernachlässigung des Dichteunterschiedes von Wasser von $4^{0}$ und Wasser von $\left.17,5^{\circ}\right)$ :

also $\quad s=0,61$.

$$
\frac{1}{1,00388}=0,99+0,01 s
$$

Während also die Zuckerlösung, was ihre Dichte anbelangt, sich wie eine Mischung von Wasser und festem Zucker verhält, ist der Einfluss auf die innere Reibung viermal grösser, als er aus der Suspendierung der gleichen Zuckermenge resultieren. würde. Es scheint mir dies Resultat im Sinne der Molekulartheorie kaum anders gedeutet werden zu können, als indem man annimmt, dass das in Lösung befindliche Zuckermolekül die Beweglichkeit des unmittelbar angrenzenden Wassers hemme, so dass ein Quantum Wasser, dessen Volumen ungefähr das. Dreifache des Volumens des Zuckermoleküls ist, an das Zuckermolekül gekettet ist.

Wir können also sagen, dass ein gelöstes Zuckermoleküł (bezw. das Molekül samt dem durch dasselbe festgehaltene Wasser) in hydrodynamischer Beziehung sich verhält wie eine Kugel vom Volumen 2,45 . 342/N $\mathrm{cm}^{3}$, wobei 342 das Molekulargewicht des Zuckers und $N$ die Anzahl der wirklichen Moleküle in einem Grammolekül ist.

\section{§ 4. Ueber die Diffusion eines nicht dissoziierten Stoffes in flüssiger Lösung.}

Es liege eine Lösung vor, wie sie in $\$ 3$ betrachtet wurde. Wirkt auf das Molekül, welches wir als eine Kugel vom Radius $P$. betrachten, eine Kraft $K$, so bewegt sich das Molekül mit einer Geschwindigkeit $\omega$, welche durch $\boldsymbol{P}$ und den Reibungskoeffi- 
zienten $k$ des Lösungsmittels bestimmt ist. Es besteht nämlich die Gleichung ${ }^{1}$ ):

$$
\omega=\frac{K}{6 \pi k P} .
$$

Diese Beziehung benutzen wir zur Berechnung des Diffusionskoeffizienten einer nicht dissoziierten Lösung. Bedeutet $p$. den osmotischen Druck der gelösten Substanz, welcher bei der betrachteten verdünnten lösung als die einzige bewegende Kraft anzusehen sei, so ist die auf die gelöste Substanz pro Volumeneinheit der Lösung in Richtung der $X$-Achse ausgeübte. Kraft. $=-\delta p / \delta x$. Befinden sich $p$ Gramm in der Volumeneinheit und ist $m$ das Molekulargewicht des gelösten Stoffes, $N$ dieAnzahl wirklicher Moleküle in einem Grammolekül, so ist $(\rho / m) \cdot N$ die Anzahl der (wirklichen) Moleküle in der Volumeneinheit und die auf ein Molekül infolge des Konzentrationsgefälles. wirkende Kraft:

$$
K=-\frac{m}{\rho N} \frac{\delta p}{\delta x} .
$$

Ist die Lösung genügend verdiunnt, so ist der osmotische Druck. durch die Gleichung gegeben:

$$
p=\frac{R}{m} \rho T,
$$

wobei $T$ die absolute Temperatur und $R=8,31 \cdot 10^{7}$ ist. Aus den Gleichungen (1), (2) und (3) erhalten wir für die Geschwindigkeit der Wanderung der gelösten Substanz:

$$
\omega=-\frac{R T}{6 \pi k} \frac{1}{N P} \frac{1}{\rho} \frac{\delta \rho}{\delta x} \text {. }
$$

Die pro Zeiteinheit durch die Einheit des Querschnittes. in Richtung der $X$-Achse hindurchtretende Stoffmenge ist endlich

$$
\omega \rho=-\frac{R T}{6 \pi k} \cdot \frac{1}{N P} \frac{\delta \rho}{\delta x} \text {. }
$$

Wir erhalten also für den Diffusionskoeffizienten $D$ :

$$
D=\frac{R T}{6 n k} \cdot \frac{1}{N P}
$$

1) G. Kirchh off, Vorlesungen über Mechanik. 26. Vorl., Gl. (22). 
Man kann also aus dem Diffusionskoeffizienten und dem Koeffizienten der inneren Reibung des Lösungsmittels das Produkt aus der Anzahl $N$ der wirklichen Moleküle in einem Grammolekül und dem hydrodynamisch wirksamen Molekularradius $P$ berechnen.

In dieser Ableitung ist der osmotische Druck wie eine auf die einzelnen Moleküle wirkende Kraft behandelt worden, was offenbar der Auffassung der kinetischen Molekulartheorie nicht entspricht, da gemäss letzterer in dem vorliegenden Falle der osmotische Druck nur als eine scheinbare Kraft aufzufassen ist. Diese Schwierigkeit verschwindet jedoch, wenn man bedenkt, dass den (scheinbaren) osmotischen Kräften, welche den Konzentrationsverschiedenheiten der Lösung entsprechen, durch ihnen numerisch gleiche, entgegengesetzt gerichtete, auf die einzelnen Moleküle wirkende Kräfte das (dynamische) Gleichgewicht geleistet werden kann, wie auf thermodynamischem Wege leicht eingesehen werden kann.

Der auf die Masseneinheit wirkenden osmotischen Kraft $-\frac{1}{p} \frac{\delta p}{\delta x}$ kann durch die (an den einzelnen gelösten Molekülen angreifende) Kraft $-P_{x}$ das Gleichgewicht geleistet werden, wenn

$$
-\frac{1}{p} \frac{\delta p}{\delta x}-P_{x}=0
$$

Denkt man sich also an der gelösten Substanz (pro Masseneinheit) die zwei sich gegenseitig aufhebenden Kräftesysteme $P_{x}$ und $-P_{x}$ angreifend, so leistet $-P_{x}$ dem osmotischen Drucke das Gleichgewicht, und es bleibt nur die dem osmotischen Drucke numerisch gleiche Kraft $P_{x}$ als Bewegungsursache übrig. Damit ist die erwähnte Schwierigkeit beseitigt.")

\section{$\S$ 5. Bestimmung der Moleküldimensionen mit Hilfe der erlangten Relationen.}

Wir haben in $\S 3$ gefunden:

$$
\frac{k^{*}}{k}=1+\varphi=1+\mathrm{n} \cdot \frac{4}{3} \pi P^{3},
$$

wobei $n$ die Anzahl der gelösten Moleküle' pro Volumeneinheit und $P$ den hydrodynamisch wirksamen Molekülradius bedeutet. Berücksichtigt man, dass

1) Eine ausflubrliche Darlegung dieses Gedankenganges findet sich in Ann. d. Phys. 17. p. 549. 1905. 


$$
\begin{aligned}
& -21- \\
& \frac{n}{N}=\frac{\beta}{m},
\end{aligned}
$$

wobei $\beta$ die in der Volumeneinheit befindliche Masse des gelösten Stoffes und $m$ dessen Molekulargewicht bedeutet, so erhält man:

$$
N P^{3}=\frac{3}{4 \pi} \frac{m}{r}\left(\frac{k^{*}}{k}-1\right)
$$

Andererseits wurde in $\$ 4$ gefunden:

$$
N P=\frac{R T}{6 \pi k} \frac{1}{D} \text {. }
$$

Diese beiden Gleichungen setzen uns in den Stand, die Grössen $P$ und $N$ einzeln zu berechnen, von welchen sich $N$ als unabhängig von der Natur des Lösungsmittels, der gelösten Substanz und der Temperatur herausstellen muss, wenn unsere Theorie den Tatsachen entspricht.

Wir wollen die Rechnung für wässerige Zuckerlösung durchführen. Nach den oben mitgeteilten Angaben über die innere Reibung der Zuckerlösung folgt zunächst für $20^{\circ} \mathrm{C}$ :

$$
N P^{3}=200 \text {. }
$$

Nach Versuchen von Graham (berechnet von $\mathrm{Stefan)}$ ist der Diffusionskoeffizient von Zucker in Wasser bei $9,5^{\circ} \mathrm{C}$. 0,384 , wenn der Tag als Zeiteinheit gewählt wird. Die Zähigkeit des Wassers bei $9,5^{\circ}$ ist 0,0135 . Wir wollen diese Daten in unsere Formel für den Diffusionskoeffizienten einsetzen, trotzdem sie an 10 proz. Lösungen gewonnen sind und eine genaue Gültigkeit unserer Formel bei so hohen Konzentrationen nicht zu erwarten ist. Wir erhalten

$$
N P=2,08 \cdot 10^{16} \text {. }
$$

Aus den für $N P^{3}$ und $N P$ gefundenen Werten folgt, wenn wir die Verschiedenheit von $P$ bei $9,5^{\circ}$ und $20^{\circ}$ vernachlässigen,

$$
\begin{aligned}
& P=9,9 \cdot 10^{-8} \mathrm{~cm}, \\
& N=2,1 \cdot 10^{23} .
\end{aligned}
$$

Der für $N$ gefundene Wert stimmt der Grössenordnung nach mit den durch andere Methoden gefundenen Werten für diese Grösse befriedigend aberein.

Bern, den 30. April 1905. 
$1906, \times 42$ 